\title{
Excited State Interaction of Laser Dyes and Silver Nanoparticles in Different Media
}

\author{
Yusif S. El-Sayed ${ }^{1,2}$, Mohamad Gaber ${ }^{1}$ \\ ${ }^{1}$ Department of Chemistry, Faculty of Science, Tanta University, Tanta, Egypt \\ ${ }^{2}$ Deanery of Academic Services, Basic Science Department, Taibah University, Madina, Saudi Arabia \\ Email: *yousifchem@yahoo.com, "yel-sayed@tu.edu.eg, "ybahnasi@taibahu.edu.sa, mabuelazm@yahoo.com
}

Received September 5, 2012; revised October 8, 2012; accepted October 24, 2012

\begin{abstract}
Quenching the fluorescence of the dyes 3-(4'-dimethylaminophenyl)-1-(1H-pyrrol-2-yl)prop-2-en-1-one (DMAPrP), 3(4'-dimethylaminophenyl)-1-(2-furanyl)prop-2-en-1-one (DMAFP) and 3-(4'-dimethylaminophenyl)-1-(2-thienyl)prop2-en-1-one (DMATP) has been investigated in the presence of silver cation and silver nanoparticles in different media. The results of the quenching experiments were analyzed using Stern-Volmer equation. Quenching the fluorescence of the dyes decreased with increasing medium viscosity. The Stern-Volmer constant $\left(K_{S V}\right)$ values in the absence and presence of ethylene glycol show that the quenching efficiencies decrease as the medium viscosity increases indicating that the quenching process is a diffusion-controlled process, and this is consistent with a dynamic-type quenching.
\end{abstract}

Keywords: Ag Nanoparticles; Laser Dyes; Surfactants, Fluorescence Quenching

\section{Introduction}

Silver nanoparticles (Ag NPs) have been a subject of great interest among scientists due to their remarkable properties such as good conductivity, catalytic and antibacterial effect [1-4]. Moreover, the successful use of Ag NPs in materials modification for application in different fields such as clothing, semiconductor and preparation of nanocomposite materials with improved performances has been demonstrated [5-7].

Fluorescence quenching is a technique to understand the interaction within the medium in view of the special role of surfaces of the nanoclusters in guiding and modifying physicochemical process. Metal nanoparticles have attracted significant attention due to their interesting optical and electronic properties, which have resulted in the exploitation of a number of applications in chemistry and biochemistry [8-13]. Fluorescence quenching of organic dyes by $\mathrm{AgCl}$ and/or Ag nanoparticles has been studied [14-16].

Chalcones and their functionalized derivatives have been shown to display diverse medical properties [17-20], interesting properties such as optical sensor [21] and electrochemical sensors for $\mathrm{Ca}$ and $\mathrm{Ba}$ [22].

In continuation of our interest in the study of photophysical properties of chalcones [23-25], the quenching of fluorescence spectra of DMAPrP, DMAFP and DMATP has been investigated by $\mathrm{Ag}^{+}$ion and $\mathrm{Ag}$ nanoparti-

"Corresponding author. cle in different media.

In the present study, we report a series of laser dyes derivatives (Scheme 1) undergo fluorescence enhancement on the interaction with Ag nanoparticles.

\section{Experimental}

\subsection{Preparation and Characterization}

The synthesis, purification and characterization of 3-(4'dimethylaminophenyl)-1-(1H-pyrrol-2-yl)prop-2-en-1one (DMAPrP), 3-(4-dimethylaminophenyl)-1-(2-furanyl)prop-2-en-1-one (DMAFP) and 3-(4-dimethylaminophenyl)-1-(2-thienyl)prop-2-en-1-one (DMATP) have been reported in a previous work [23-25] as follow into a conical flask $250 \mathrm{ml}$ equipped with magnetic stirring were placed 5.5 g sodium hydroxide $(\mathrm{NaOH})$

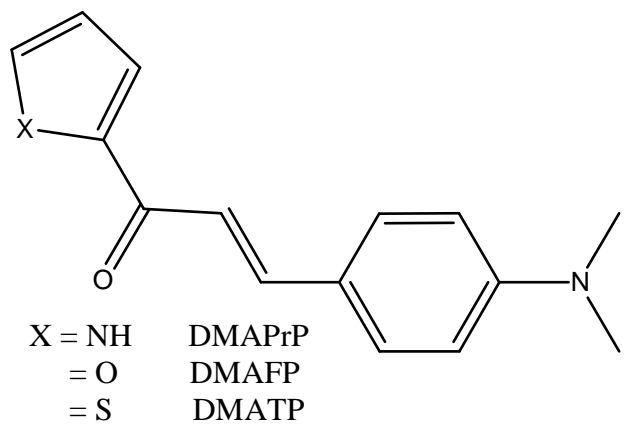

Scheme 1. Series of laser dyes (chalcones) derivatives under investigation. 
( $0.01375 \mathrm{~mol})$ dissolved in $50 \mathrm{ml}$ of second distilled water and $0.01 \mathrm{~mol}$ ketone compounds dissolved in $25 \mathrm{ml}$ of ethanol (EtOH). Then $0.01 \mathrm{~mol}$ of 4-N,N-dimethyl amino benzaldehyde dissolved in $25 \mathrm{ml} \mathrm{EtOH}$ was added dropwise. The mixture was stirred at $30^{\circ} \mathrm{C}$ (progress of reaction was monitored by Thin-Layer Chromatography (TLC) using ethyl acetate/petroleum ether as eluent $(1: 9 \mathrm{v} / \mathrm{v}))$. After $6 \mathrm{~h}$, the product precipitated from the solution. After cooling in a refrigerator overnight, the solid product was filtered, washed with water and 95\% ethanol, dried under vacuum and further purified by recrystallization from ethanol, yielding $70 \%$ of the product.

$\mathrm{AgNO}_{3}$ purchased from Aldrich was used without further purification. Deionized water was used throughout the work. Sodium dodocyle sulfate (SDS), cetyltrimethyl ammonium bromide (CTAB) from BDH, triton $\mathrm{x}-100$ (TX-100) from Aldrich were used without further purifycation.

The fluorescence spectra of the dyes with $\mathrm{Ag}^{+}$ion and Ag nanoparticles as quenchers were recorded using Shimadzu RF 510 spectrofluorometer.

Correction of the emission spectra response was performed using the method of Melhuish [26-28] using $10^{-5}$ $\mathrm{M}$ solution of anthracene in benzene (originally reported in quanta by measuring absolute intensities of emitted photons at various wavelengths as a reference standard; $\lambda_{e x}=365 \mathrm{~nm}$ ). From the observed and corrected emission spectra of $10^{-5} \mathrm{M}$ solution of anthracene in benzene the correction factor at each wavelength was calculated then applied to correct the present emission spectra. In quenching studies, because of the absorption of the excitation and the emission light by the quencher, correction for both of these was performed using the method described by Marciniak [29] that applied the following equations:

$$
I_{f}^{c o r r}=I_{f}^{o b s} \frac{\varepsilon_{D} c_{D}+\varepsilon_{Q} c_{Q}}{\varepsilon_{D} c_{D}}\left[\frac{1+10^{-\varepsilon_{D} c_{D}{ }^{l}}}{1-10^{-\left(\varepsilon_{D} c_{D}+\varepsilon_{Q} c_{Q}\right)^{l}}}\right]
$$

where $I_{f}^{c o r r}$ and $I_{f}^{o b s}$ are fluorescence intensities corrected and observed for the absorption of exciting light by the quencher, respectively, $\varepsilon_{D}$ and $\varepsilon_{Q}$ are the molar absorption coefficient at the exciting wavelength for donor and quencher, respectively, $c_{D}$ and $c_{Q}$ are molar concentrations of donor and quencher, respectively and $l$ is the exciting wavelength.

$$
I_{f}^{\prime c o r r}=\frac{I_{f}^{o b s}}{10^{-\varepsilon_{Q}^{\prime} c_{Q}^{i}}}
$$

where $I_{f}^{o b s}$ and $I_{f}^{\text {corr }}$ are fluorescence intensities observed and corrected for the absorption of exciting light by the quencher, respectively, $\varepsilon_{Q}$ is the molar absorption coefficient of the quencher at the emission wavelength monitored, and $I^{\prime}$ is the effective path length for reabsorption of fluorescence.

Absorption of the solutions was measured by a Shimadzu UV-160A spectrophotometer. Transmission electron microscopy (TEM) was carried out on a model JEOL JEM-100SX Electron Microscope with a field gun, and an accelerating voltage of $20 \mathrm{kV}$. The images were recorded at different magnifications from 500 to 3500 at different time intervals. Samples were prepared by placing and drying a drop of solution on a copper grid coated with a thin amorphous carbon film.

\subsection{Preparation and Characterization of $\mathrm{Ag}$ Nanoparticles}

Before the preparation, all the glassware must be soaked in aqua regia, and then washed by water several times and dried. The preparation steps are follows, into a clean conical bottle containing $40 \mathrm{~mL}$ water, $0.385 \mathrm{ml}$ of $2.4 \times$ $10^{-2} \mathrm{~mol} / \mathrm{L}$ silver nitrate $\left(\mathrm{AgNO}_{3}\right)$ and $3.5 \mathrm{~mL}$ of $1.0 \%$ sodium citrate solution were added under the stirring, 4 $\mathrm{mL}$ of $0.05 \%$ sodium borohydride $\left(\mathrm{NaBH}_{4}\right)$ solutions were added slowly. After $10 \mathrm{~min}$, it was diluted to 50.0 $\mathrm{mL}$ with water [30]. Transmission electron micrographs and UV-Vis spectra were then recorded to characterize the prepared Ag nanoparticles.

The silver nanoparticles have the maximum absorption peak at $405 \mathrm{~nm}$ and the average size was $15 \mathrm{~nm}$. Different volumes of this solution have been used to quench the fluorescence of DMAPrP, DMAFP and DMATP.

\subsection{Calculations of Silver Nanoparticles Parameters}

Since one begins with $1 \times 10^{-3} \mathrm{M} \mathrm{AgNO}_{3}$ in making the silver nanoparticles one has a concentration of silver nanoparticles if we assumed that all $\mathrm{Ag}^{+}$ions are reduced by the reducing agent, sodium borohydride $\mathrm{NaBH}_{4}$.

Silver atom has cubic close-packed fcc crystal structure with cell parameters $\mathrm{a}=\mathrm{b}=\mathrm{c}=4.0853 \AA$. In an fcc unit cell, the corner atoms account for $(1 / 8) \times 8=1$ atom, and those in the center of the faces for $(1 / 2) \times 6=3$. The fcc unit cell contains four atoms with volume = $68.182333 \AA^{3}$. In both the hcp and fcc structures voids account for only $25.96 \%$ of the total volume [31].

Diameter of silver nanoparticle $=150 \AA$. Volume of the silver nanoparticle is $1.8 \times 10^{6} \AA^{3}$ and the number of silver atoms per particle is $1.056 \times 10^{5}$ atoms/particle. The number of silver atoms is $5.6 \times 10^{21}$ per $50 \mathrm{~mL}$, so the number of particles is $1.054 \times 10^{18}$ per liter. The concentration of Ag nanoparticles in mole per liter is $1.8 \mathrm{~nm}$. For silver nanoparticles of $0.995 \mathrm{~nm}$ concentration, the optical density was 1.0945 OD at $\lambda_{\max }=405 \mathrm{~nm}$, the extinction coefficient $(\varepsilon(\lambda))$ of the prepared silver nanoparticles is $1.1 \times 10^{9} \mathrm{M}^{-1} \cdot \mathrm{cm}^{-1}$. 


\section{Results and Discussion}

\subsection{Characterization of the Prepared Ag Nanoparticles}

The size distributions of the metal particles synthesized in this work have been analyzed by high resolution transmission electron microscopy (HRTEM) images, showing that at least $80 \%$ of the particles are distributed around $15 \mathrm{~nm}$. Figure 1 shows the electronic absorption spectra of different concentrations of Ag-nanoparticles in aqueous solution. Typical concentrations are (a) 0.199, (b) 0.398 and (c) $0.587 \mathrm{~nm}$. The insert in Figure 1 shows the HRTEM image of Ag-nanoparticles of about $15 \mathrm{~nm}$ diameter. The absorption band of Ag nanoparticles shows an absorption maximum at $405 \mathrm{~nm}$. The HRTEM image shows that the prepared particles are spherical and of about $15 \mathrm{~nm}$ diameter.

\subsection{Emission of Laser Dyes in the Presence of Nanoparticles}

Figure 2 shows the fluorescence spectra of $10 \mu \mathrm{M}$ DMAPrP (the spectra DMAFP and DMATP are not shown here) methanol: water (1:1) by volume in presence of variable concentrations of $\mathrm{Ag}^{+}$ion and/or $\mathrm{Ag}$ nanoparticles. The effect of surfactants on the fluorescence quenching process was measured at the critical micellar concentration (cmc) of SDS, Triton X-100 and $\mathrm{CTAB}$ as anionic, neutral and cationic micelles, respecttively. Also, the effect of medium viscosity on the fluorescence quenching process was cheeked by adding various amounts of ethylene glycol $(20 \%$ and $50 \%$ by volume). The emission spectra of DMAPrP, DMAFP and

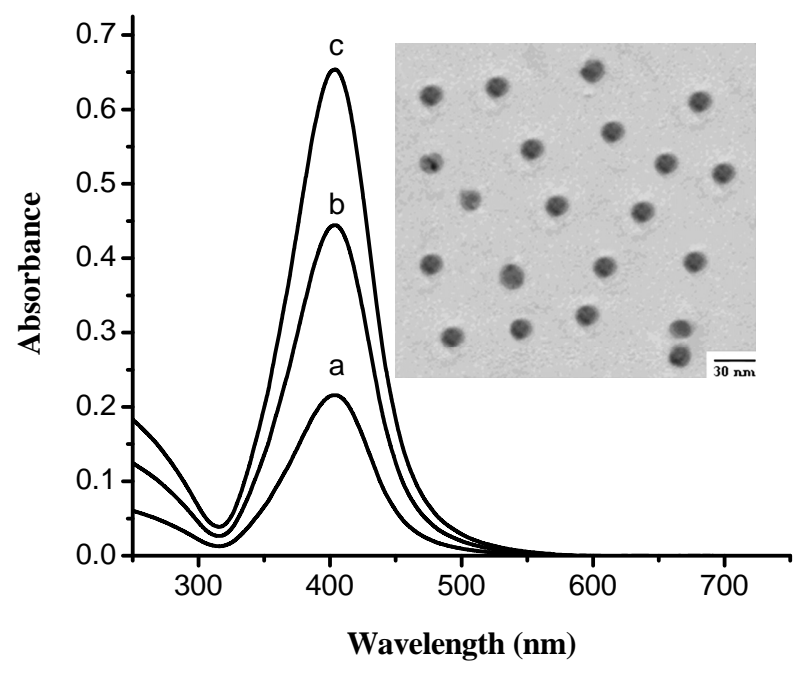

Figure 1. Absorption spectra of Ag-nanoparticles in aqueous solution of concentration (a) 0.199 , (b) 0.398 and (c) $0.597 \mathrm{~nm}$. The inserted shows the TEM image of Ag-nanoparticles of about $15 \mathrm{~nm}$ diameter.

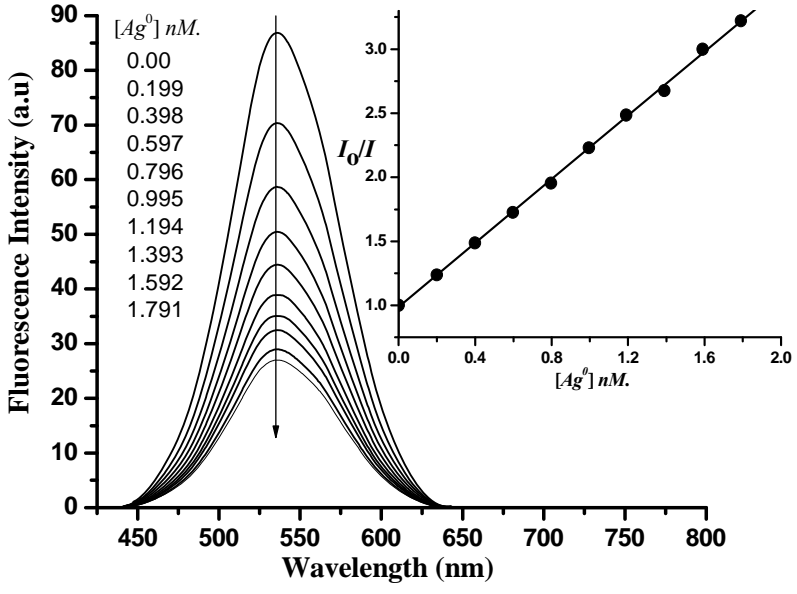

Figure 2. Enhanced fluorescence emission of DMAPrP (10 $\boldsymbol{\mu M})$ as a result of the addition of $\mathrm{Ag}$ nanoparticles (concentration indicated on the figure) at $\lambda_{e x}=370 \mathrm{~nm}$ and $\lambda_{e m}=$ $535 \mathrm{~nm}$.

DMATP in the media were mentioned above exhibit a maximum at 535, 544 and $551 \mathrm{~nm}$, respectively.

As the concentration of $\mathrm{Ag}^{+}$ion and/or Ag nanoparticles quencher increases, the position of the fluorescence bands does not change despite of the substantial decrease in the fluorescence intensities. This indicates the absence of significant molecular interactions under the prevailing experimental conditions.

Under the experimental conditions, the excitation wavelength of $370 \mathrm{~nm}$ is away from the maximum absorption of the Plasmon resonance peak of Ag nanoparticles by ca $35 \mathrm{~nm}$ and the Ag nanoparticles don't exhibit any fluorescence. In addition, there is no quenching of laser dyes under investigation emission caused by citrate ions indicating that $\mathrm{Ag}$ nanoparticles are responsible for this effect.

It is well known that metallic surface induces strong quenching of molecular fluorescence due to electromagnetic coupling between the metal and the fluorescent molecule [32].

The quenching follows the Stern-Volmer relation $I_{o} / I=$ $1+K_{S V}[Q]$, where $I_{o}$ and $I$ are the emission intensities of dyes in absence and presence of quencher $\left(\mathrm{Ag}^{+}\right.$ions and/or Ag nanoparticles) of concentration $[Q]$, respecttively, and $K_{S V}$ is the Stern-Volmer quenching constant. The Stern-Volmer plots of the quenching process of DMAPrP (plots of DMAFP and DMATP are not shown here) emission by Ag nanoparticles in absence and presence of ethylene glycol are shown in Figure 3, which shows that the quenching efficiencies decrease as the medium viscosity increases indicating that the quenching process is a diffusion-controlled process, and this is consistent with a dynamic-type quenching.

Also, the quenching efficiencies in the micellar media follow the order TX-100 > CTAB $>$ SDS agreement with 


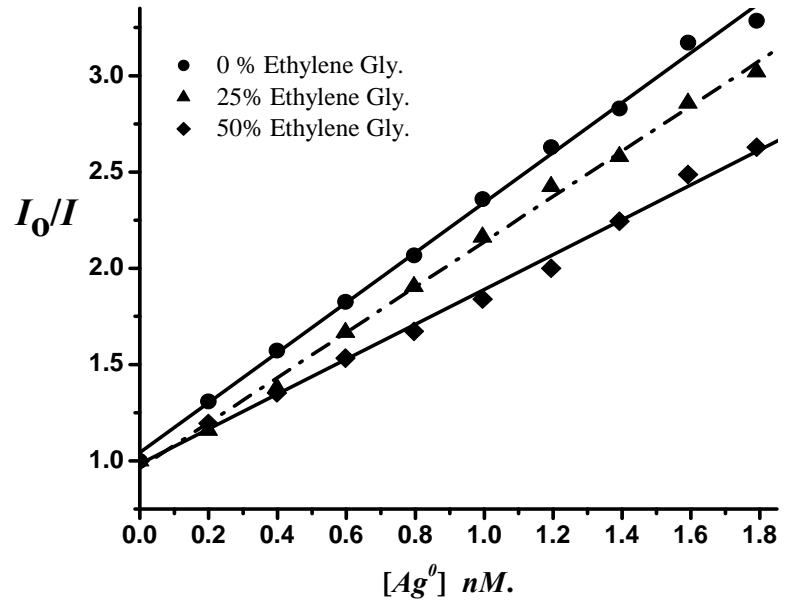

Figure 3. Stern-Volmer plots for fluorescence quenching of $10 \mu M$ DMAPrP by Ag nanoparticles in different ratios of ethylene glycol (viscosity effect) at room temperature.

the viscosity of medium.

The Stern-Volmer plots deviate from the linearity at higher concentration of the quencher (Ag nanoparticles), this may be due to the aggregation of Ag nanoparticles upon increasing their concentrations which will increase the possibility of static quenching relative to the dynamic type quenching prevailing at lower concentrations of nanoparticles.

The values of $K_{S V}$ were calculated in all media and given in Table 1. It is shown that, Ag nanoparticles quench the fluorescence of dyes under investigation with extraordinary high Stern-Volmer constants $\left(K_{S V}\right)$ in the range of $10^{9} \cdot \mathrm{M}^{-1}$.

These $K_{S V}$ values remarkably high quenching constants are not common in small molecular quenchers; these values are 7 - 8 orders of magnitude larger than small dye molecules quenchers [33]. This extraordinary quenching ability is rationalized in terms of the number of surface silver atoms per each silver particle of $15 \mathrm{~nm}$ diameter is $9 \times 10^{3}$ atoms. The corresponding to $10^{4}$ surface sites, assuming one surface site per surface Ag atom, as a result, we can assume that the fluorescence emission of 10 $\mu \mathrm{M}$ dye molecules may be quenched by each Ag nanoparticle.

\subsection{The Quenching Mechanism}

It is now well established that the binding of probe molecules to the metal surface of the nanoparticles results in quenching of the excited state [34]. Both energy transfer and electron transfer processes are considered to be the major deactivation pathways for excited fluoroprobes on the metal surface. The electron transfer mechanism is predominant for particle sizes of $<5 \mathrm{~nm}$ as the particles don't exhibit any surface plasmon band in the visible region $[33,35]$. The degree of quenching de- pends on the structural details that control proximity between the fluorophore and the metal nanoparticles core and since the metal particles are larger than $5 \mathrm{~nm}$, energy transfer dominates the quenching mechanism [32].

Since we have $15-\mathrm{nm}$ diameter silver nanoparticles, resonant energy transfer between the donor and acceptor takes place when a donor molecule is placed in the vicinity of a conductive metal surface. The probability of this Froster energy transfer depends on the overlap of the normalized emission band of the probe molecule with the extinction spectrum of the silver nanoparticles. Figure 4, illustrates that the excitation spectrum of the Ag nanoparticles overlaps significantly with the laser dyes emission, giving efficient energy transfer with a calculated critical transfer distance $\left(R_{0}\right)$ equal 62.8, 56.5 and $52 \AA$ for Ag NPs-DMAPrP, DMATP and DMAFP pairs, respectively.

A number of investigations have been carried out in improving our understanding of the phenomenon of solubilization by surfactant solutions [36-39]. The main interest concerns the extent to which a particular compound can be solubilized in a given surfactant solution at a specified concentration.

The other important aspect is to know the regions where the solubilizate molecules locate within the micelles. There are different ways in which a solubilizate can arrange itself within the micelles; it can either arrange itself on the micellar surface, or it can be incorporated in the micellar core or it can be at the interface of the core as shown in Scheme 2.

So, this process reduces the fluorescence quenching. Also, the fluorescence quenching by metallic nanoparticles affected by the absence and presence of micelles, the way for solubilization may be arranged by a different way around the nanoparticles due to their highly adsorbed characters, see Scheme 3.

The extent of solubilization and the site occupied by the fluorophore molecules depend upon the structural and chemical nature of both the surfactant and the solubilizate as well as the concentration of the species in solution and the temperature. The presence of the solubilizate can affect the process of micellization and affect the thermodynamics of the micellization by affecting the interactions.

\section{Conclusions}

With increase in the concentration of Ag nanoparticles, the laser dyes under investigation were adsorbed effectively and the fluorescence of the laser dyes was quenched. The quenching of the fluorescence of laser dyes by metallic Ag nanoparticles in 1:1 (methanol:water) solution may also occur by the adsorption of the laser dyes on the surface of nanoparticles, where $K_{S V}$ values 
Table 1. Stern volmer quenching constants by $\mathrm{Ag}$ nanoparticles and $\mathrm{Ag}^{+}$ion.

\begin{tabular}{|c|c|c|c|c|c|c|}
\hline \multirow{3}{*}{ Medium } & \multicolumn{6}{|c|}{$K_{S V^{*}} M^{-1}$} \\
\hline & \multicolumn{2}{|c|}{ DMAPrP } & \multicolumn{2}{|c|}{ DMATP } & \multicolumn{2}{|c|}{ DMAFP } \\
\hline & $\mathrm{Ag}^{0} \times 10^{-7}$ & $\mathrm{Ag}^{+}$ & $\mathrm{Ag}^{0} \times 10^{-7}$ & $\mathrm{Ag}^{+}$ & $\mathrm{Ag}^{0} \times 10^{-7}$ & $\mathrm{Ag}^{+}$ \\
\hline $\mathrm{MeOH}$ & 128 & 1.36948 & 120 & 1.20081 & 116 & 1.16224 \\
\hline SDS & 121 & 1.20513 & 118 & 1.17824 & 114 & 1.14012 \\
\hline СТАВ & 120 & 1.20029 & 114 & 1.14472 & 109 & 1.09396 \\
\hline TX-100 & 116 & 1.16224 & 113 & 1.13059 & 111 & 1.10871 \\
\hline 25\% Gly & 119 & - & 114 & - & 109 & - \\
\hline 50\% Gly & 91 & - & 84 & - & 78 & - \\
\hline
\end{tabular}

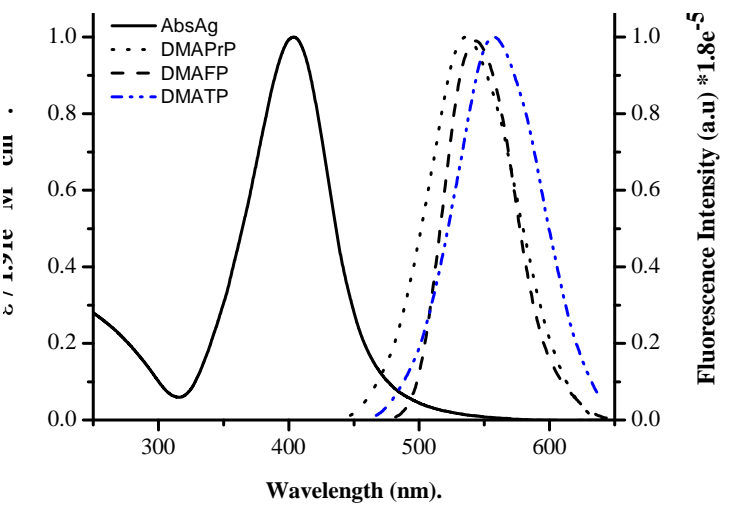

Figure 4. Normalized absorption spectra of $0.597 \mathrm{~nm}$ silver nanoparticles and normalized emission spectrum of $10 \mu \mathrm{M}$ DMAPrP, DMAFP and DMATP in methanol: water (1:1) mixed solvent. The calculated value of $R_{0}$ for silver nanoparticles-DMAPrP, DMATP and DMAFP pairs were $R_{0}=$ 62.8, 56.5 and $52 \AA$, respectively.

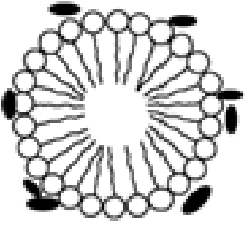

(a)

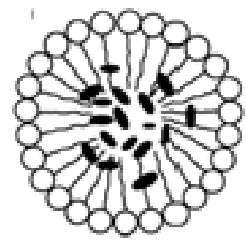

(b)

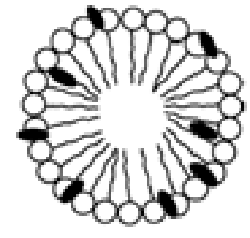

(c)
Scheme 2. Different regions within the micelle where the fluorophore molecules locate itself (a) on the surface, (b) in the micelle core, (c) at the interface.

are also high. The Stern Volmer quenching constant by $\mathrm{Ag}^{+}$ion in 1:1 (methanol:water) solution is very low indicating absence of adsorption of the dye molecules on the quencher surface.

The quenching of the fluorescence of laser dyes by $\mathrm{Ag}^{+}$ in aqueous medium also suggests that the electrostatic effect between the cationic dye and cationic quencher $\left(\mathrm{Ag}^{+}\right)$ may be responsible for the reduced quenching. The

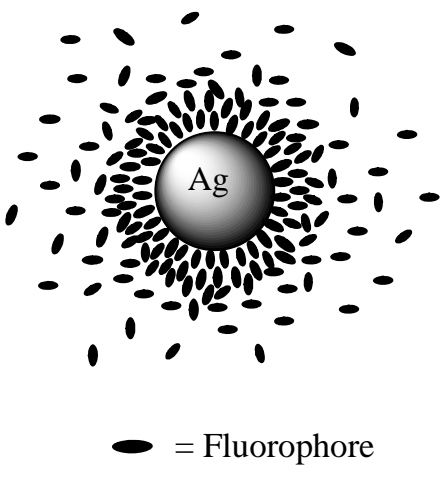

(a)

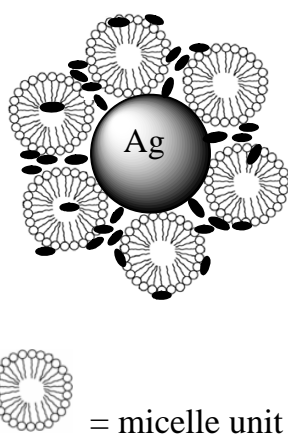

(b)
Scheme 3. The adsorption process on the metallic nanoparticle surface in a) absence and $b$ ) presence of micelles.

process has been found to be less efficient (with signifycantly low $K_{S V}$ values) in 1:1 (methanol:water) solution.

\section{Acknowledgements}

The authors are deeply indebted to the team work at Tanta University for their valuable help and critical guidance and for facilitating many administrative procedures. This research work was financed and supported-by Tanta University, Tanta, Egypt.

\section{REFERENCES}

[1] J. M. Kohler, L. Abahmane, J. Wagner, J. Albert and G. Mayer, "Preparation of Metal Nanoparticles with Varied Composition for Catalytical Applications in Microreactors," Chemical Engineering Science, Vol. 63, No. 20, 2008, pp. 5048- 5055. doi:/10.1016/j.ces.2007.11.038

[2] Z. L. Jiang, C. Y. Liu and L. W. Sun, "Catalytic Properties of Silver Nanoparticles Supported on Silica Spheres,” The Journal of Physical Chemistry B, Vol. 109, No. 5, 2005, pp. 1730-1735. doi:/10.1021/jp046032g

[3] K. H. Cho, J. E. Park, T. Osaka and S. G. Park, "Optimization of the Sputter-Deposited Platinum Cathode for a Direct Methanol Fuel Cell,” Electrochimica Acta, Vol. 51, 
No. 5, 2005, pp. 956-960. doi:/10.1016/j.electacta.2005.04.071

[4] [4] A. Petica, S. Gavriliu, M. Lungu, N. Buruntea and C. Panzaru, "Colloidal Silver Solutions with Antimicrobial Properties.” Materials Science and Engineering: B, Vol. 152, No. 1-3, 2008, pp. 22-27.

[5] P. Gupta, M. Bajpai and S. K. Bajpai, "Investigation of Antibacterial Properties of Silver Nanoparticle-Loaded Poly (Acrylamide-co-Itaconic Acid)-Grafted Cotton Fabric,” Journal of Cotton Science, Vol. 12, No. 3, 2008, pp. 280-286.

[6] P. D. Cozzoli, R. Comparelli, E. Fanizza, M. L. Caurri, A. Agostiano and D. Laub, "Photocatalytic Synthesis of Silver Nanoparticles Stabilized by $\mathrm{TiO}_{2}$ Nanorods: A Semiconductor/Metal Nanocomposite in Homogeneous Nonpolar Solution,” Journal of the American Chemical Society, Vol. 126, No. 12, 2004, pp. 3868-3879. doi:/10.1021/ja0395846

[7] L. Armelao, G. Bottaro, R. Campstrini, S. Gialanella and M. Ischia, "Synthesis and Structural Evolution of Mesoporous Silica-Silver Nanocomposites," Nanotechnology, Vol. 18, No. 15, 2007, p. 155606. doi:/10.1088/0957-4484/18/15/155606

[8] A. P. V. Kamat, "Photophysical, Photochemical and Photocatalytic Aspects of Metal Nanoparticles,” The Journal of Physical Chemistry B, Vol. 106, No. 32, 2002, pp. 7729-7744. doi:/10.1021/jp0209289

[9] J. R. Lakowicz, "Radiative Decay Engineering: Biophysical and Biomedical Applications," Analytical Biochemistry, Vol. 298, No. 1, 2001, pp. 1-24. doi:/10.1006/abio.2001.5377

[10] J. R. Lakowicz, Y. Shen, S. D’Auria, J. Malicka, J. Fang, Z. Gryczynski and I. Gryczynski, "Radiative Decay Engineering: 2 Effects of Silver Island Films on Fluorescence Intensity, Lifetimes, and Resonance Energy Transfer," Analytical Biochemistry, Vol. 301, No. 2, 2002, pp. 261-277. doi:/10.1006/abio.2001.5503

[11] J. R. Lakowicz, "Radiative Decay Engineering 3 Surface Plasmon-Coupled Directional Emission,” Analytical Biochemistry, Vol. 324, No. 2, 2004, pp. 153-169. doi:/10.1016/j.ab.2003.09.039

[12] K. Aslan, I. Gryczynski, J. Malicka, J. R. Lakowicz and C. D. Geddes, "Metal-Enhanced Fluorescence: An Emerging Tool in Biotechnology," Current Opinion in Biotechnology, Vol. 16, No. 1, 2005, pp. 55-62. doi:/10.1016/j.copbio.2005.01.001

[13] K. Aslan, P. Holley, L. Davies, J. R. Lakowicz and C. D. Geddes, "Angular-Ratiometric Plasmon-Resonance Based Light Scattering for Bioaffinity Sensing," Journal of the American Chemical Society, Vol. 127, No. 34, 2005, pp. 12115-12121. doi:/10.1021/ja052739k

[14] A. Al-Kady. S, M. Gaber, M. M. Hussein and E. M. Ebeid, "Fluorescence Enhancement of Coumarin Thiourea Derivatives by $\mathrm{Hg}^{2+}, \mathrm{Ag}^{+}$, and Silver Nano- particles.” Journal of Physical Chemistry A, Vol. 113, No. 34, 2009, pp. 9474-9484. doi:/10.1021/jp905566z

[15] S. Pramanik, S. C. Bhattacharya and T. Imae, "Fluo- rescence Quenching of 3,7-Diamino-2,8-dimethyl-5-ph- enyl
Phenazinium chloride by AgCl and Ag nano- particles," Journal of Luminescence, Vol. 126, No. 1, 2007, pp. 155-159. doi:/10.1016/j.jlumin.2006.06.008

[16] I. S. Lee and H. Suzuki, "Quenching Dynamics Promoted by Silver Nanoparticles,” Journal of Photochemistry and Photobiology A: Chemistry, Vol. 195, No. 2-3, 2008, pp. 254-260. doi:/10.1016/j.jphotochem.2007.10.009

[17] M. A. Babu, N. Shakya, P. Prathipati, S. G. Kaskhedikar and A. K. Saxena, "Development of 3D-QSAR Models for 5-Lipoxygenase Antagonists: Chalcones,” Bioorganic \& Medicinal Chemistry, Vol. 10, No. 12, 2002, pp. 4035-4041. doi:/10.1016/S0968-0896(02)00313-9

[18] S. Kumar, E. Hager, C. Pettit, H. Gurulingappa, N. E. Davidson and S. R. Khan, "Design, Synthesis, and Evaluation of Novel Boronic-Chalcone Derivatives as Antitumor Agents,” Journal of Medicinal Chemistry, Vol. 46, No. 14, 2003, pp. 2813-2815. doi:/10.1021/jm030213+

[19] M. Liu, P. Wilairat, C. L. Simon, A. L. Tan and M. Go, "Structure-Activity Relationships of Antileishmanial and Antimalarial Chalcones," Bioorganic \& Medicinal Chemistry, Vol. 11, No. 13, 2003, pp. 2729-2738. doi:/10.1016/S0968-0896(03)00233-5

[20] M. S. Karthikeyan, B. S. Holla and N. S. Kumari, "Synthesis and Antimicrobial Studies on Novel Chloro-Fluorine Containing Hydroxy Pyrazolines,” European Journal of Medicinal Chemistry, Vol. 42, No. 1, 2007, pp. 30-36. doi:/10.1016/j.ejmech.2006.07.011

[21] T. A. Fayed, “A novel Chalcone-Analogue As An Optical Sensor Based on Ground and Excited States Intramolecular Charge Transfer: A Combined Experimental and Theoretical Study," Chemical Physics, Vol. 324, No. 2-3, 2006, pp. 631-638. doi:/10.1016/j.chemphys.2005.11.039

[22] B. Delavaux-Nicot, J. Maynadie, D. Lavabre and S. Fery-Forgues, " $\mathrm{Ca}^{2+}$ vs. $\mathrm{Ba}^{2+}$ Electrochemical Detection by Two Disubstituted Ferrocenyl Chalcone Chemosensors. Study of the Ligand-Metal interactions in $\mathrm{CH}_{3} \mathrm{CN}$," Journal of Organometallic Chemistry, Vol. 692, No. 4, 2007, pp. 874-886.

[23] M. Gaber, S. A. El-Daly, T. A. Fayed and Y. S. Y. El-Sayed, "Photophysical Properties, Laser Activity and Photoreactivity of a Heteroaryl Chalcone: A Model OF Solvatochromic Fluorophore,” Optics \& Laser Technology, Vol. 40, No. 3, 2008, pp. 528-537. doi:/10.1016/j.optlastec.2007.08.006

[24] S. A. El-Daly, M. Gaber, Y. S. El-Sayed, "Photophysical Parameters and Laser Performance of 3-(4'-Dimethylaminophenyl)-1-(2-Furanyl)prop-2-en-1-One (DMAFP): A New Laser Dye," Optics \& Laser Technology, Vol. 41, No. 6, 2009, pp. 727-733.

[25] Y. S. El-Sayed, S. A. El-Daly, M. Gaber, "Spectral Behavior and Laser Activity of 3-(4'-Dimethylamino phenyl)1-(1H-pyrrol-2-yl)prop-2-en-1-one (DMAPrP). A New Laser Dye,” Optics \& Laser Technology, Vol. 42, No. 2, 2010, pp. 397-402.

[26] W. H. Melhuish, “Modified Technique for Determining the Wavelength-Sensitivity Curve of a Pectrofluorimeter," Applied Optics, Vol. 14, No. 1, 1975, pp. 26-27. 
[27] W. H. Melhuish, "Calibration of Spectrofluorimeters for Measuring Corrected Emission Spectra," The Journal of the Optical Society of America, Vol. 52, No. 11, 1962, p. 1256. doi:/10.1364/JOSA.52.001256

[28] W. H. Melhuish. "A Standard Fluorescence Spectrum for Calibrating Spectro-Fluorophotometers,” Journal of Physical Chemistry, Vol. 64, No. 6, 1960, pp. 762-764. doi:/10.1021/j100835a014

[29] B. Marciniak, "Does Cu(acac)2 Quench Benzene Fluorescence?: A Physical Chemistry Experiment,” Journal of Chemical Education, Vol. 63, No. 11, 1986, pp. 998-1000. doi:/10.1021/ed063p998

[30] W. Cai, Y. Fan, Z. Jiang and J. Yao, “A Highly Sensitive and Selective Resonance Scattering Spectral Assay for Potassium Ion Based on Aptamer and Nanosilver Aggregation Reactions," Talanta, Vol. 81, No. 4-5, 2010, pp. 1810-1815. doi:/10.1016/j.talanta.2010.03.046

[31] P. C. Lee and D. Meisel, "Adsorption and Surface-Enhanced Raman of Dyes on Silver and Gold Sols," Journal of Physical Chemistry, Vol. 86, No. 17, 1982, pp. 33913395. doi:/10.1021/j100214a025

[32] S. K. Ghosh, A. Pal, S. Kundu, S. Nath and T. Pal, "Fluorescence Quenching of 1-Methylaminopyrene near Gold Nanoparticles: Size Regime Dependence of the Small Metallic Particles," Chemical Physics Letters, Vol. 395, No. 4-6, 2004, pp. 366-372. doi:/10.1016/j.cplett.2004.08.016

[33] C. Fan, S. Wang, J. W. Hong, G. C. Bazan, K. W. Plaxco and A. J. Heeger, "Beyond Superquenching: Hyper- Efficient Energy Transfer from Conjugated Polymers to Gold Nanoparticles," Applied Physics, Vol. 100, No. 11, 2003, pp. 6297-6301.

[34] P. Avouris and B. N. J. Persson, "Excited States at Metal Surfaces and Their Non-Radiative Relaxation,” Journal of Physical Chemistry, Vol. 88, No. 5, 1984, pp. 837-848. doi:/10.1021/j150649a004

[35] B. I. Ipe, K. G. Thomas, S. Barazzouk, S. Hotchandani and P. V. Kamat, "Photoinduced Charge Separation in a Fluorophore-Gold Nanoassembly,” Journal of Physical Chemistry B, Vol. 106, No. 1, 2002, pp. 18-21. doi:/10.1021/jp0134695

[36] S. K. Mehta, N. Bala and S. Sharma, "Thermodynamics of Aggregation of Tweens in the Presence of Diclofenac Sodium,” Colloids and Surfaces A, Vol. 268, No. 1-3, 2005, pp. 90-98. doi:/10.1016/j.colsurfa.2005.06.013

[37] V. Suratkar and S. Mahapatra, "Solubilization Site of Organic Perfume Molecules in Sodium Dodecyl Sulfate Micelles: New Insights from Proton NMR Studies," Journal of Colloid and Interface Science, Vol. 225, No. 1, 2000, pp. 32-38. doi:/10.1006/jcis.2000.6718

[38] V. J. Sovilj and L. B. Petrovic, "Influence of Molecular Characterissssstics of Nonionic Cellulose Ethers on Their Interaction with Ionic Surfactant Investigated by Conductometry," Colloid \& Polymer Science, Vol. 284, No. 3, 2005, pp. 334-339. doi:/10.1007/s00396-005-1376-4

[39] L. Ceraulo, F. Filizzola, A. Longo, A. Ruggirello and V. T. Liveri, "Physicochemical Investigation of the SolubiLization of Ytterbium Nitrate in AOT Reverse Micelles and Liquid Crystals,” Colloid \& Polymer Science, Vol. 284, No. 10, 2006, p. 1485. doi:/10.1007/s00396-006-1485-8 